\title{
Setting Up Of A Writing Process In Academic Context To Produce Meaning In French
}

\author{
Assia Benabid, Assistant Prof. \\ Casablanca hassan ii university faculty of science ben’ msik casablanca \\ doi: 10.19044/esj.2016.v12n23p390 URL:http://dx.doi.org/10.19044/esj.2016.v12n23p390
}

\begin{abstract}
Today pedagogy's main preoccupation lies in focusing on postgraduate students' writing in science classes. This is the case for two reasons. The first reason takes into account student's difficulties in acquiring linguistic competences. Therefore, the overall curriculum has to allow more time for this activity. The second reason is related to the evolution in the pedagogical discourse, for it insists on the active role that students should benefit from during their language learning. It is in this way that they can learn to become efficient. To train science students in Morocco in understanding and producing a postgraduate scientific discourse in French requires a preparation, which will provide them with some clues regarding their writing ability. Indeed, by installing a writing process enhancing the building up of meaning, these students will become capable of deciphering and producing scientific discourse.
\end{abstract}

Keywords: Language and terminology, scientific discourse, meaning, writing process

\section{Introduction}

L'intérêt des recherches en didactique des sciences pour les pratiques d'écriture n'est pas nouveau. En effet, l'émergence des travaux en didactique de l'écriture a eu lieu au milieu des années quatre-vingt et vient en relais aux travaux concernant la lecture.

Durant ces années, on a observé aussi une évolution générale de la conceptualisation des pratiques scolaires d'écriture. Celle-ci est influencée essentiellement par la question du sens de ces pratiques (Ecrire pour qui ? écrire pourquoi faire?).

Les principales difficultés rencontrées au niveau de la langue française par les étudiants scientifiques, nous ont motivé et donc incité à faire la présente recherche à travers laquelle nous avons réfléchi au processus d'écriture. Dans notre contexte universitaire, on a remarqué que très rares 
sont les professeurs qui proposent par exemple des travaux de réécriture à leurs étudiants.

Par ailleurs, lors d'entretiens les professeurs affirment s'appuyer davantage sur l'oral que sur l'écrit. La question est alors d'étudier les conditions d'accompagnement de ces étudiants dans leur processus de réécriture. Dans cet article, nous avons limité volontairement le développement de la compétence écrite (compréhension écrite et production écrite) à la mise en place d'un processus d'écriture chez les étudiants scientifiques sans prendre en compte les recherches concernant la compréhension de l’écrit.

\section{I.}

Module Langue et terminologie : Quelles pratiques d'écrit dans la classe de langue à l'université marocaine?

La terminologie est actuellement l'objet d'un module enseigné à l'université marocaine. Le décideur a vu pertinent d’intégrer un module de « Langue et Terminologie » dans les deux premiers semestres des cursus de formation.

Cette décision est l'effet d'une remise en question « peu délibérée » du module transversal Langue et Communication.

Dès lors, le cours de LT fait partie d'un bloc de modules complémentaires, dont le volume horaire représente 15\% à 25\%du volume horaire de la filière.

L’attribution du statut de « module complémentaire » au cours de « Langue et Terminologie » suscite alors plusieurs questionnements :

Le statut « complémentaire » est-il synonyme de « transversal »? c’est bien le cas, ce module devrait donc servir d'accompagnement linguistique et disciplinaire aux étudiants pour leur permettre une immersion dans les autres contenus de la formation disciplinaire au sein de l'université.

La relation syntaxique de coordination qui anime les deux objets « langue » et

« terminologie » est source de flous didactiques (le pôle du savoir), d’où l’émergence de certaines questions préoccupantes :

Que devrions - nous alors enseigner?

La langue et la terminologie ensemble?

La langue française pour elle-même et la terminologie pour ellemême ?

La langue de spécialité (langue disciplinaire) ?

Les termes de la discipline?

Le discours scientifique?

Autres objets de savoirs ? Reste à préciser. 
En revanche, organiser du sens dans une spécialité en français ne consiste pas en la seule capacité à maîtriser un lexique pointu. Ce dernier, prend en fait place dans des discours proposant à la fois une démarche et des intentions qui justifient une élaboration textuelle spécifique concernant à la fois explication et argumentation. Le discours scientifique universitaire par exemple est à la fois un discours de connaissances et de reconnaissance proposant une recherche et une méthodologie devant être validées par des pairs et s'éloignant de la compilation encyclopédique. Or, l'étudiant scientifique limité par le temps dont il dispose pour mener à bien ses études, élabore un certain nombre de priorités, quitte à réduire ses difficultés à un problème lexical au détriment d'une approche des discours universitaires dont les exigences académiques ne lui sont pourtant pas familières et sans l'apprentissage desquelles il risque de se sentir véritablement et culturellement exclu par des implicites trop prégnants.

En effet, le module Langue et Terminologie constitue ainsi une déclinaison Français sur Objectif Spécifique pour étudiants, organisée autour de savoir-faire devant rendre compte d'une recherche en cours. Ces savoirfaire doivent permettre par exemple au sens de s'élaborer au-delà du mot et de la phrase tout en offrant au niveau du texte un maximum d'informations dans un minimum de mots de manière claire et concise.

Ainsi, les ateliers de reformulation par exemple proposent pour leur part des activités permettant de :

- $\quad$ Planifier, hiérarchiser, assurer la progression et la continuité du sens

- $\quad$ Élaborer un questionnement

- $\quad$ Chasser l'ambiguïté (anaphores : précision lexicale et pronominale)

- $\quad$ Supprimer l’inutile (pléonasmes, phrases creuses, redondance)

- $\quad$ Insister sur la lecture et la relecture au bénéfice d'une réécriture concise.

Néanmoins, les activités pluridisciplinaires peuvent par ailleurs donner l'occasion aux étudiants de réaliser des dossiers personnels. Mais, là encore ces écrits sont considérés comme des résultats et la plupart du temps, ils ne donnent pas lieu à un travail langagier pour apprendre les sciences. C'est un peu encore comme si l'écriture était transparente, sauf dans les cas où un professeur de français participe au projet et engage les étudiants à des activités de réécriture.

Cependant, dans la classe la rédaction d'un texte scientifique intervient après une recherche documentaire dans le domaine par exemple des biotechnologies, accompagnée de discussions de groupes et la consigne d'écriture est de rédiger un texte explicatif, du type de ceux des manuels scolaires, à destination d'étudiants. Or, l'écrit a une place importante dans l'enseignement des sciences. Mais, le plus souvent, les écrits produits par les étudiants sont des écrits d'exposition (Vérin, 1988 ; Astolîi\& al.,1991), 
rédigés au terme d'un travail et répondant à des normes précises : comptes rendus d'expériences, réponse à des questions, résumés.

\section{L’écriture : l'écriture d'expression et l'écriture expositive}

L'écriture d'expression est une écriture d'investigation utilisée comme outil au service de la production d'explications ; elle permet à l'étudiant d'explorer sa pensée personnelle et de la clarifier en matérialisant et couchant "sur le papier" les relations entre ses connaissances. Les étudiants sont engagés dans une écriture à la première personne, dans le sens où c'est leur propre pensée qui est en jeu et non des connaissances scientifiques qui existeraient ici comme extérieures à l'apprenant (Vérin, 1995,1999). Cette forme d'écrit est informelle, car les étudiants sont encouragés à utiliser leur propre langage (Prain\& Hand, 1996 b ; Shawn \&al., 1994).

Cependant, contrairement à l'écriture expositive qui permet rarement aux étudiants l'engagement mental nécessaire à la production de sens (Holliday\&al., 1994), cette forme d'écrit leur permet de s'expliquer plus clairement sur les concepts scientifiques; les idées comprises par les étudiants prennent du sens et sont donc plus facilement mémorisables et applicables (Prain\&Hand, 1996 b ; Shawn \& al., 1994).

L'écriture d'expression est donc une écriture pour comprendre et pour apprendre. C'est pourquoi l'essentiel des travaux de didactique concernant les pratiques d'écriture en cours de sciences s'intéresse à l'écriture d'expression, laquelle s'impose à la fin de cette dernière décennie comme le paradigme dominant. La pratique de l'écriture d'expression repose sur la prise en compte des processus cognitifs du scripteur lors du processus rédactionnel qui a pu être modélisé.

Dans le cadre de l'écriture d'expression, les productions des étudiants constituent "une fenêtre sur leurs processus de pensée" qui permet non seulement aux chercheurs mais aussi aux enseignants et aux étudiants euxmêmes d'observer la construction de sens qui s'effectue lors du travail rédactionnel. Du fait de la constitution d'un produit matérialisé, l'écriture facilite une mise à plat des idées. La pratique de l'écriture d'expression implique donc de sortir de la logique de l'écriture expositive et demande dès lors, un nouvel examen des pratiques didactiques. Dans le paragraphe cidessous nous allons étudier les conditions d'accompagnement de ces étudiants scientifiques dans leur processus d’écriture.

\section{Processus d'écriture : Avant- texte}

Les conceptions courantes de l'apprentissage par l'écriture restent encore embryonnaires et fragmentées. L'écrit constitue une "mémoire de papier", permettant un retour sur les traces antérieures (mémoire à long terme) et déchargeant la gestion mentale simultanée de plusieurs 
informations (mémoire de travail). Bien qu'ancienne, la modélisation de Bereiter et Scardamalia (1987) reste la pierre angulaire de nombreuses recherches des années quatre-vingt-dix (Holliday\& al. 1994 ; Keys, 1999). Bereiter et Scardamalia ont proposé deux modèles du processus de composition : le modèle d'expression des connaissances et le modèle de transformation des connaissances. Le premier modèle correspond à l'écriture expositive, le second à l'écriture interprétative plus souvent désignée sous le terme d'écriture d'expression. Ce deuxième modèle permet d'expliquer qu'en produisant un écrit qui lui sert à construire des interprétations (dimension herméneutique), un étudiant est capable de transformer ses connaissances. L'écriture, peut ainsi permettre de construire du sens et favorise les changements conceptuels.

En effet, l'expression des connaissances est la réponse habituelle pour générer du contenu dans un discours prenant la forme mentale de la tâche à accomplir et la génération des thèmes d'écriture ; il y a appropriation des tâches quotidiennes d'écriture, mais pas génération de nouvelles connaissances. Dans les prochains paragraphes, nous allons présenter les étapes du processus d'écriture :

\section{L'écriture $\longrightarrow$ Réecriture $\longrightarrow$ SENS $\longrightarrow \quad$ Les aléas de l'écriture $\longrightarrow$ Texte final}

Le travail de l'écriture en train de se constituer par exemple ; par le recours au manuscrit c'-à-d l'accès aux variantes nous permet de saisir le sens dans le mouvement, voire dans les variations que quand il est figé, l’écriture se présente alors plus comme une activité qu' un art figé.

Toutefois, le texte est par nature indécidable irrémédiablement disponible, pluriel puisque toute lecture analyse met en mouvement des relations ou associations imprévues, inespérées voire prémonitoires. Il y a donc des hésitations, l'étudiant est indécis, avance, recule car il se trouve au moment où sa relation à la langue est plus visible. Ce travail est à la recherche du sens, recherche sémantique, l'on tiendra compte que la réécriture refoule un certain langage pour laisser en émerger un autre. Dans ce va et vient va se manifester ce qui est l'étudiant par rapport à la langue. C'est bien par les tentations et les sollicitations de plusieurs voies sémantiques qu’on retrouve le sens.

En revanche, tout est révélateur pour l'étudiant, une biffure, une substitution, une permutation peuvent altérer le sens on peut observer que le sens est présent partout quels que soient les circuits, réseaux ainsi que les parcours empruntés même les blocages, les difficultés linguistiques, les vides ont un sens, le sens appelle donc le sens. C'est pourquoi on peut interroger le texte pour montrer comment l'écriture dans son mouvement fait retour sur elle-même afin de créer son propre espace au point que la linéarité se transforme en spirale, multipliant les effets de sens. 
Ainsi, le sens n'est sûrement pas dans la fixité mais dans la dynamique des altérations de la production écrite. En effet, le texte définitif est le résultat de nombreux textes préalables. Toute production écrite ne peut naître toute conçue. Pour frayer un chemin vers son sens et son expression, le discours traverse un milieu d'expression et d'accents étrangers, il est à l'unisson avec certains de ses éléments, en désaccord avec d'autres. Dans ce processus de dynamisme, il donne alors forme à son image et à son tour stylistique.

Cependant, on aurait tendance à croire que l'infini des possibles changements s'annule avec la finitude du texte scientifique. Mais rien n'est sûr ni certain car à tout moment se présentent des changements voire des transformations qui font dévier le cours des choses et compromettent ainsi l'accomplissement initial du texte.

Le texte ne cesse de se modifier à travers les réécritures donc de nouvelles lectures se profilent, certains éléments paragraphes, phrases, verbes, mots peuvent ainsi être éliminés et constituent ce qu'on appelle des impasses.

En effet, les impasses montrent que le désordre de l'esprit est créateur et qu'il existe un autre type d'ordre sous-jacent qui échappe même au créateur. Comme dans l'élaboration du rêve qui est une problématique de reviviscence. Si l'on veut tenir compte de toute la valeur du mot, une qualité nouvelle entre enfin « les mots devenus vivants débordent de toute part et veulent s'assembler en dessins dans lesquels la précision ne suffit plus ». Selon Ernst Jünger "graffiti" Frontalières 10/18 p 119 Paris, 1977.

La notion de réécriture est donc employée pour désigner l'opération de révision de la première version d'un texte. En procédant à des transformations qui donnent un caractère nouveau, le texte est rectifié et donc différent du premier. On peut prêter à la notion de

" réécrire » une valeur méta-discursive. Ainsi la problématique de l'écriture est suggérée par le biais des variantes qui pose le problème d'un sens nouveau introduit dans la variation.

En effet un texte s'efface au profit d'un autre qui recueille toute la tension dramatique du texte abandonné, ceci n'est pas sans nous rappeler l'écrivain en train d'écrire son texte, il choisit alors ses mots, recherche constamment le terme propre, exact, adéquat. Il essaie de traquer le mot le plus juste, celui qui conviendrait le mieux. Il tâte et soupèse les mots afin de les élire ou bien les rejeter, selon qu'ils traduisent ou non ce qu'il a vraiment voulu dire. Ainsi, finit par se former une phrase digne de ce que cette dernière a voulu décrire dans le rythme, la syntaxe, la ponctuation, créant ainsi un ensemble unique offert aux autres. L'approximation fait place à la stylisation progressive, c'est-à-dire à la réaction en chaine du sens. 
A ce propos, Michel Leiris soutient que l'écrivain doit affronter le risque d'écrire comme le toréador affronte le risque des cornes du taureau. La métaphore qu'utilise Leiris est très révélatrice et significative à la fois, car elle montre combien l'écrit reste très subtil et délicat en lui-même puisqu'il pénètre nos pensées. Parfois le stylo ou la plume peuvent soudainement ralentir en pleine écriture, ils ne glissent plus sur la feuille mais raclent le fond du dictionnaire afin de trouver le mot qui couvrira de son apparence anodine une pensée qui peut s'avérer être dangereuse. En effet, le stylo ou la plume retiennent nos pensées, les corrigent et les traitent comme des minéraux bruts jusqu'à l'obtention des mots polis. Le texte s'écrit alors dans cette tension d'une recherche qui se dérobe.

Il faut souligner que chaque recherche intellectuelle est donc caractérisée par des "errances d'esprit». Il y a un moment de " jeu» pendant lequel l'intelligence tâtonne et se propose plusieurs solutions au problème qui la préoccupe. On peut donc comparer l'écriture à une longue et lente rêverie ou longue hésitation devant la feuille blanche, un griffonnage involontaire et inconscient se produit. Une turbulence et une errance d'esprit qui ne cessent de se torturer afin d'aboutir à trouver la tournure qui coincide avec un état d'approximation mentale.

Nous ressentons alors un malaise à bâcler les choses, c'est un labeur cérébral éreintant puisqu'on cherche les mots qui voudront bien s'accorder et s'harmoniser avec notre pensée. Nous recherchons avec volupté le vocable radieux qui se meurt d'attente derrière le vague, la brume, là peu près où notre pensée oscille. Cette opération ne se fait pas sans difficulté. Nous pouvons en déduire que c'est dans ce jeu de mot l'écrivain ou toute personne qui écrit sélectionne ; refuse et insiste quelque chose alors vient au jour qui ne peut se dire que là ; ce qui ne va certainement pas sans angoisse ni contrecoups sur la personne en train d'écrire son texte.

Cette dimension du devenir est intéressante en soi car l'activité des mots nous est donnée à voir, il suffit d'effacer, de rayer, de changer, de substituer, ou d'éliminer pour que tout s'altère et change, il n'y a pas de forme fixe ni statique car tout peut se réélaborer ; dévoilant alors d'autres aspects, d'autres possibilités de la langue qui vont renouveler et faire perdurer une culture. En évoquant ce mouvement perpétuel qu'on peut appeler circularité ou réminiscence apparaît que la « fin » n'existe pas que tout peut recommencer c'est dire que l'écrit reste une chose mouvante ; ductile et modulable à l'infini et c'est bien par le biais des variantes qui expriment le doute, par rejet, retour, reprise ou ajout qu'on aperçoit l'invention de l'écriture, la réécriture n'est donc pas fortuite et ne résulte nullement d'un hasard, bien au contraire il y a une élaboration tissée.

Ainsi, ce désordre s'avère être un principe énergétique indispensable et nécessaire qui finit par donner des résultats concrets et positifs. 
En outre, la reformulation est une pratique langagière d'objectivation de l'objet et de la pratique. Tous les discours mis en œuvre dans les situations scientifiques font appel à la pratique de reformulation qui nous paraît fondamentale dans la construction des savoirs.

En effet, comme pour toute production langagière la première opération consiste à ancrer le discours dans un contexte pertinent, ce qui suppose de négocier le sens de l'activité. La difficulté à résoudre est multiforme, et se cristallise à la fois dans la nature du discours et dans le registre langagier de l'énoncé à adopter.

Le sens se cherche par reformulation et partant, par réécriture, il y a donc une dynamique qui le produit et se crée, dans la mesure où l'on produit du sens en faisant éclater le sens premier, donc en le ravivant. Ceci amène à dire qu'il n'y a pas de forme fixe que le langage est donc instable et peut à tout moment être changé, réapproprié. On défait quelque chose de stable afin d'y loger autre chose qui ne peut se dire que là.

C’est un « séisme » de sens qui apparaît, et avec toutes ces altérations qui s'opèrent, quelque chose vient au jour.

En utilisant la nature des variantes, tout laisse à penser qu'il y a un texte avec plusieurs formulations alors qu'en réalité, il n'existe que deux textes. Dans un article Jean Peytard l'a exprimé avec force à propos de Lautréamont : adoptant « comme hypothèse que la correction ne s'élabore pas sur le détail ou sur une succession de détails en cours d'une relecture, comme on ébarbe une épreuve d'imprimerie de ses coquilles, mais dans le projet d'ensemble, une pratique de déconstruction-construction qui façonne un texte autre Jean Peytard " Lautréamont la nouvelle critique n 37, paris, 1970, p 44 ».

Ainsi la volonté de changer ne suffit certes pas pour assurer un changement du moins peut-elle être considérée comme étant une amorce voire une incitation en vue d'une réorientation, d'un infléchissement.

De plus, ce qui demeure important c'est bien la variation des états c'est aussi la confrontation d'un texte avec tous les possibles qui le composent en amont comme en aval, c'est enfin la mobilité complexe et la stabilité précaire des formes. Il s'agit de porter un regard sur cette textualité mouvante de tous les instants qui n'est autre que l'activité d'écriture et de pensée aux prises avec la visée d'un texte, d'un projet qui se veut l'affirmation d'une expression de plus en plus personnelle.

\section{La quête du sens : Construction de sens et changement conceptuel}

Il faut essayer de maîtriser au mieux ce travail différentiel. Ce jeu de compensation dans lequel une tension de divergence se crée entre texte de départ et texte final et une dynamique s'installe. On refuse, on taille, on élimine, on revient sur le mot, on le change pour en garder un autre en 
remplacement ceci laisse apercevoir, la quête du sens qui n’est autre que dans la variation et c'est là, où il tarde à se fixer qu'il s’exprime le plus.

Les tâches d'écriture pourraient être utiles à l'apprentissage dans les cas où un contact entre auteur et lecteur est organisé au cours du processus d'écriture, ce qui révèle que les besoins et attentes respectifs de l'un et de l'autre soient plus systématiquement pris en compte.

Si l'on veut évaluer les écrits terminaux, il faut sortir du modèle du compte rendu expérimental et aborder des genres d'écriture différents, ce qui ne veut pas dire accepter un niveau insuffisant. En effet, les élèves peuvent élaborer leur compréhension des concepts scientifiques en utilisant leur propre langage, mais dans une formulation scientifiquement acceptable. La scientificité des écrits d'expression des étudiants ne repose pas obligatoirement sur l'utilisation de genres spécifiques ou de vocabulaire technique. S'ils utilisent le style narratif, les étudiants doivent montrer que tous les détails de l'histoire (ses composantes, son fil conducteur, les caractères des personnages et les situations), concrétisent leur compréhension des concepts clés concernant le sujet étudié (Prain\& Hand, 1996 b ; Sutton, 1993, 1995).

En fait, la scientificité des textes des étudiants peut être évaluée au cours des travaux successifs de réécriture qui permettent aux étudiants d'améliorer leurs productions écrites. Celle-ci est repérable par la production d'inférences spécifiques qui expliquent la signification des données, et des justifications ("preuves"). Les étudiants ne s'engagent pas tous de la même manière dans l'écriture scientifique ni avec la même force argumentative ; mais dans tous les cas l'appropriation du processus argumentatif reste partiel (Kelly \& Chen, 1999 ; Keys, \&al., 1999).

La maîtrise de l'écriture du genre argumentatif plus tardive que celle du genre narratif ne semble pas signifier que la maîtrise de ce genre est plus difficile, mais est plutôt dû à un enseignement plus restreint de ce genre (Brassard, 1990). Il est possible d'aider les étudiants à mieux maîtriser le processus argumentatif en les initiant aux pratiques du discours scientifique. On peut par exemple leur faire étudier des articles qui serviront de référence ; ainsi Moore (1994 a) propose de faire découvrir aux étudiants l'importance de la production d'arguments, par la lecture d'un article de vulgarisation et l'analyse des procédés discursifs présents dans celui-ci. Selon Martin (1999), Veel (1996) et d'autres auteurs, les stratégies les plus efficaces pour que les étudiants acquièrent les règles et les significations des différentes pratiques langagières en science sont les suivantes : l'analyse détaillée des caractéristiques linguistiques d'exemples textuels, la construction de genres avec leur professeur et enfin l'explicitation extensive par le professeur des caractéristiques textuelles clefs et de leur raison d'être. 
L'enseignant de langue, à diverses reprises, incite les étudiants à se référer aux écrits produits au cours des différentes étapes, comme par exemple les hypothèses et l'expérience proposée ou encore les résultats de l'expérience et les hypothèses, pour qu'ils s'efforcent de les mettre en correspondance : "Vous allez reprendre les hypothèses que vous avez notées et je vais vous demander de bien regarder cette hypothèse et de bien reréflechir/je dirai à une expérience qui permette de vraiment vérifier l'hypothèse. " [Consigne donnée par l'enseignant de langue lors d'un cours de langue et terminologie].

Les résultats de ce processus montrent que les étudiants ont développé des stratégies textuelles pour expliquer des concepts et des procédés et ils ont même développé leur processus d'écriture. Pour expliquer les résultats du processus d'écriture des étudiants et leur perception de la tâche, plusieurs facteurs peuvent être identifiés comme potentiellement importants :

Les connaissances préalables des étudiants sur le sujet et leur attitude par rapport à celui-ci;

$>\quad$ La nature spécifique de l'exercice écrit;

$>\quad$ Le rôle des activités de conception comprenant la rédaction des premières versions et le travail de révision;

Les connaissances des étudiants sur la manière d'aborder des problèmes de rédaction tels que l'organisation du contenu et la prise en compte des besoins et attentes du lecteur.

\section{Conclusion}

L'analyse didactique du rôle de l'écrit et surtout la production du sens dans le processus d'écriture pour les apprentissages dans des domaines scientifiques spécifiés est encore embryonnaire, cet article ouvre donc cette voie qui paraît tant féconde que prometteuse.

Développer la compétence écrite chez les étudiants scientifiques implique une diversification des productions écrites en cours des sciences. Il est indispensable de ne plus se cantonner à des écrits expositifs terminaux aux caractéristiques normées (tel le traditionnel compte rendu) mais plutôt d'envisager des écrits exploratoires courts et non notés (Astolfi\&Ducancel., 1995 ; Vérin, 1995). Contrairement à ce qui se passe au Maroc, dans les pays anglo-saxons par exemple, la diversification est très importante : pièces de théâtre, scripts de slogan, recettes, récits, guides, poésies, cartes conceptuelles, posters, brochures, biographies, journaux, texte humoristique... (Audet\&al., 1996 ;Burcke, 1995 ; Duran \& al.,1998; Henderson \& Wellington, 1998; Hildebrand, 1998; Prain\& Hand, 1995, 1996, 1999 ; Sutton, 1992, 1995). Il y a donc différents types de tâches d'écriture visant le développement de la compétence écrite chez les étudiants 
scientifiques. Par contre, les concepts scientifiques sont construits dans un langage et une organisation souvent totalement opposés au langage et aux concepts quotidiens.

Ainsi, il serait légitime de se poser la question suivante : y a t-il de la place pour un processus d'écriture reliant les deux sens : langage spécialisé et langage quotidien, explications et apprentissage scientifique ?

Cette recherche conduit ainsi à proposer des questions pour les futures recherches portant cette fois-ci sur la conception de tâches d'écriture pour apprendre que des séquences d'enseignement favorisent ce processus d'écriture.

\section{References:}

Christian Orange, Jean-Claude Fourneau, Jean-Paul Bourbigot, écrits de travail, débats scientifiques et problématisation à l'école élémentaire : http://ife.ens-lyon.fr/publications/edition-

electronique/aster/ASTER_2001_33_111.pdf

Thierry Lebeaupin Centre de Linguistique Appliquée Université de FrancheComté, Objectifs universitaires en français : langue-culture, médiation des savoirs et recherche.

Quelles clefs pour l'étudiant étranger ? http://gerflint.fr/Base/Monde8T1/lebeaupin.pdf

Pierre Fillon Anne Vérin, écrire pour comprendre les sciences, http://ife.enslyon.fr/publications/edition-electronique/aster/RA033.pdf

Laurence Catel, écrire pour apprendre ? écrire pour comprendre ? État de la question http://ife.ens-lyon.fr/publications/editionelectronique/aster/ASTER_2001_33_17.pdf

Assia BENABID, La Variance Textuelle Dans " La Naissance Du Jour" de Colette, Processus d'écriture, Année 2003 THESE (SENS ) 